\title{
Epidural Anesthesia to Effectively Manage Pain and Facilitate Rehabilitation in a Pediatric Case of Complex Regional Pain Syndrome
}

\author{
Goran Tubic, MD
}

There is a paucity of information related to treatment of pediatric CRPS. Treatment of CRPS in pediatric patients has been guided by adult recommendations, which consist of a multidisciplinary approach involving pharmacotherapy, physical therapy, and psychotherapy, as appropriate. Patients unable to tolerate physical therapy with traditional oral pharmacotherapy may require more invasive pain management techniques such as sympathetic blocks, epidural infusion of analgesics, or spinal cord stimulation to facilitate restoration of function.

This case report describes the successful use of epidural infusion of fentanyl, clonidine, and bupivacaine through a tunneled epidural lumbar catheter for pain management in an 11-year-old girl who developed complex regional pain syndrome I (CRPS I) approximately 2 months after sustaining an injury to her right knee. Following short-lasting pain relief from 3 repeated blocks, she underwent an implant of a tunneled epidural catheter (TEC) and a 4-week infusion of fentanyl (2 $\mathrm{mcg} / \mathrm{mL})$, clonidine $(1 \mathrm{mcg} / \mathrm{mL})$, and bupivacaine $(0.04 \%)$. At last follow-up, approximately 3.5 months after implant of the TEC, the patient's pain and symptoms were completely resolved, her range of motion and function were completely restored, and her physical activity had returned to pre-injury levels.

Key words: Complex regional pain syndrome (CRPS), tunneled epidural catheter, pediatric, continuous regional anesthesia, epidural analgesia, continuous epidural anesthesia, interventional pain management
The International Association for the Study of Pain (IASP) uses the term complex regional pain syndrome (CRPS) to describe localized conditions characterized by spontaneous or evoked pain disproportionate in severity and duration to the usual clinical course of the originating trauma or injury (1). The most notable symptoms of CRPS include extreme pain in the form of hyperalgesia or allodynia; swelling and/or changes in skin color of the affected limb; dry, mottled skin; muscle weakness; and sensory loss. Currently, there is no specific test or set of tests that can definitively diagnose CRPS; a diagnosis is reliant on physical exam and medical history and is largely one of exclu-

Author for correspondence: Goran Tubic, MD

Address: Chicagoland Pain Management, 420 S. Schmidt, Ste 110, Bolingbrook, IL 60440

E-mail: gtubic@hotmail.com sion. According to the Budapest clinical diagnostic criteria for CRPS (2), 4 criteria must be met to confirm a diagnosis. These include 1) continuation of pain that is disproportionate to the initial injury or event; 2 ) at least one symptom in 3 out of 4 categories including sensory, vasomotor, sudomotor/edema, or motor/ trophic; 3) at least one clinical sign at the time of evaluation in 2 or more categories including sensory (hyperalgesia or allodynia), vasomotor (temperature asymmetry and/or skin color changes and/or asymmetry), sudomotor/edema (edema and/or sweating changes and/or sweating asymmetry), or motor/trophic (decreased range of motion, weakness, tremor, dystonia, trophic changes in hair, nail, or skin); and 4) all other diagnoses have been ruled out. Careful assessment is needed to ensure that the patient's symptoms are properly aligned with a diagnosis of CRPS and not that of another disorder. Magnetic resonance imaging (MRI), bone scans, x-rays, and 
thermography or other procedures are useful in ruling out other conditions.

CRPS has been designated as a rare disease by the National Organization for Rare Disorders (NORD), with fewer than 200,000 cases per year in the United States (3). CRPS is even more rare in pediatric patients, with less than $10 \%$ of all cases occurring during childhood and adolescence (4). Consequently, there is a paucity of information to inform treatment in this patient population. Treatment of CRPS in pediatric patients has been guided by adult recommendations aimed at reducing pain and restoring function in the affected limb. These recommendations include a multidisciplinary approach consisting of pharmacotherapy, physical therapy, and psychotherapy, as appropriate (1). Patients unable to tolerate physical therapy with traditional oral pharmacotherapy may require more invasive pain management techniques such as sympathetic blocks, epidural infusion of analgesics, or spinal cord stimulation to facilitate restoration of function. A recent literature review suggests that successful management of CRPS requires more invasive interventional measures in up to $35 \%$ of children (5).

We present the case of an 11-year-old girl successfully treated with epidural infusion of fentanyl $(2 \mathrm{mcg} /$ $\mathrm{mL}$ ), clonidine (1 $\mathrm{mcg} / \mathrm{mL})$, and bupivacaine $(0.04 \%)$ through a tunneled epidural lumbar catheter after developing a severe case of complex regional pain syndrome I (CRPS I) in her right knee. The purpose of this case report is to inform clinicians of a reliable, highly successful method for managing pain and facilitating rehabilitation in pediatric CRPS cases where other methods have failed.

\section{CASE PRESENTATION}

The patient and her authorized guardian provided written consent to publish this case report. An 11-year-old girl was referred to our pain management clinic for evaluation and management of pain in her right knee approximately 2 months after sustaining an injury while playing soccer. The patient was seen by an orthopedic specialist the day after the injury, presenting with pain and swelling, moderate effusion and tenderness over the medial joint line and medial femoral condyle, but with no erythema or warmth in the knee. Pain intensity was rated a 6 on a 10-point scale and she described the pain as sharp with worsening during extension and weight-bearing. Radiographic imaging revealed no fracture or dislocation and MRI showed no evidence of tendinopathy or fracture, only trace joint effusion and marrow edema along the lateral aspect of the medial femoral condyle. By the time the patient was seen in our clinic, her pain had both worsened and spread both above and below the knee to her groin and foot and she was unable to walk without the aid of crutches. She had been seen previously by 6 different physicians who had been unable to help her, with some implying exaggeration of pain symptoms, and was in significant emotional distress evidenced by constant crying. The patient now rated her pain an 8 on a 10-point scale and was unable to tolerate her knee being touched or extended. Examination of the knee revealed swelling, coldness, a darkening in color, sweating, and allodynia. A diagnosis of complex regional pain syndrome type 1 (CRPS 1 ) was made based on the patient's history and physical examination according to the Budapest clinical diagnostic criteria.

The patient failed to receive sufficient pain relief with topiramate (50 mg daily) and had only limited pain relief with lumbar sympathetic ganglion blocks (LSGB) at L2/L3. I had to complete these blocks in the operating room because of the patient's extreme anxiety and distress around having her knee touched. The patient had attempted physical therapy, but the therapy aggravated her knee pain, rendering her unable to make successful gains in therapy. We were forced to explore more advanced interventional pain management procedures due to the minimal relief from the LSGBs coupled with the severe amount of anxiety and mental block the patient had regarding her knee. The decision was made to insert an epidural catheter for infusion of local anesthetic over the course of 4 weeks to facilitate rehabilitation.

After intravenous delivery of antibiotics and induction of general anesthesia through endotracheal intubation, the patient was placed in the prone position and the surgical site was sterilized. The L4-L5 interspace was identified under $\mathrm{x}$-ray guidance and lidocaine $(0.5 \%)$ was applied to the skin and subcutaneous tissue. A 3.5-inch, 18-gauge epidural Tuohy needle was used to access the epidural space using the loss 
of resistance technique and an epidural catheter with stylet was advanced to the right side, next to the L3 neural foramina. An epidurogram with contrast solution showed excellent local spread over the nerve roots at L2-L4, indicating an ideal position for the epidural catheter for long-term infusion. A 5 -inch, 18-gauge epidural Tuohy needle was then used to tunnel the catheter approximately 5 inches in the direction of the left side from the original insertion point. The needle was then removed, the incision closed, and the catheter exit point was secured. The catheter was loaded with $5 \mathrm{~mL}$ of $2 \%$ lidocaine with epinephrine (1 to 200,000) and infusion of the epidural medication consisting of fentanyl $(2 \mathrm{mcg} /$ $\mathrm{mL})$, clonidine $(1 \mathrm{mcg} / \mathrm{mL})$, and bupivacaine $(0.04 \%)$ was instated at a continuous rate of $1.5 \mathrm{~mL} / \mathrm{hr}$. The patient was examined for sensory and motor function after successful extubation and transferred to the recovery room. Upon examination, she had partial sensory and motor block, allowing full examination of her right knee without pain for the first time since presenting to the clinic.

The patient was placed on prophylactic antibiotic therapy for the duration of tunneled epidural catheter placement and was seen in the office every 7 days for sterile dressing changes and by home health twice a week for at-home management of the epidural catheter. The epidural medication was titrated to a continuous infusion rate of $2.5 \mathrm{~mL} / \mathrm{hr}$ with a $1 \mathrm{~mL}$ bolus every 30 minutes as needed one week after the procedure. The following week, the clonidine was removed from the epidural solution and the rate was dropped back down $1.5 \mathrm{~mL} / \mathrm{hr}$. Three days later, the infusion rate was decreased to $1 \mathrm{~mL} / \mathrm{hr} ; 3$ days after that, the rate was further dropped to $0.5 \mathrm{~mL} / \mathrm{hr}$.

The catheter was removed in the office 4 weeks after placement. The patient tolerated the treatment well with minimum side effects, reporting only initial itchiness on her stomach, which was resolved with oral diphenhydramine, and minor upset stomach and nausea after clonidine was removed from the epidural solution.

Physical therapy was reinstated the day after implantation of the tunneled epidural catheter, initially starting with mirror therapy and graded motor imagery techniques such as pictures of her walking and run- ning. The Recognise ${ }^{\mathrm{TM}}$ mobile application, which has been shown to assist with rehabilitation in a range of complex pain states when used with graded motor imagery programs, was utilized. The patient attended physical therapy up to 5 times a week. Within the first 20 sessions ( 4 weeks), the patient was able to take steps without her crutches and fully straighten her knee. By the end of 12 weeks, she was able to run and jump, albeit with some slight coordination issues and some minor sensitivity to touch. Physical therapy was supplemented with psychotherapy up to 3 times per week.

At last follow-up in our clinic, approximately 3.5 months after implant of the tunneled epidural catheter, the patient's pain and symptoms were completely resolved, her range of motion and function were completely restored, and her physical activity had returned to pre-injury levels.

\section{DISCUSSION}

In the current case, the patient had been dismissed and undertreated by 6 physicians prior to arriving at our clinic. Unfortunately, it is not unusual for CRPS patients, especially pediatric patients, to be made to feel that the pain is all in their head or for their pain intensity to be discredited or minimized - despite the fact that the pain associated with CRPS has been ranked higher than childbirth and amputation. This likely is a result of limited information, as well as reluctance and inexperience with this patient population on the part of health care providers. CRPS is rare in pediatric patients, with less than $10 \%$ of all cases occurring during childhood and adolescence (4). Consequently, there is a paucity of information available to clinicians concerning the management of CRPS in pediatric patients, specifically regarding more invasive interventional options. Two literature reviews concerning the use of invasive interventional procedures in the management of CRPS in the pediatric population were published in 2015 (5-6). Both reviews summarized the evidence for use of epidural catheters, but failed to identify any randomized controlled studies in which conservative management was compared to more invasive interventions despite these more advanced interventions being required for successful pain and symptom management in up to $35 \%$ of all patients (5). Epidural drug infusion 
with local analgesics is a viable alternative when conventional treatments such as exercise and physical therapy do not achieve desired results, or in situations like the current case where the patient is unable to tolerate physical therapy. The benefits of epidural drug infusion in terms of being able to quickly adjust dosage, the multitude of potential drug combinations, and minimization of complications and risks through careful drug titration and adequate education make it a very attractive treatment option. This approach has been used successfully for management of CRPS in many adult patients, often providing much more powerful pain relief than other options. Although used infrequently in pediatric patients in general, our practice has recently started using epidural drug infusion in our pediatric patients with great success. No guidance on epidural drug infusion parameters in pediatric patients currently exists. The parameters we use for epidural drug infusion are largely based on those which have been successful in our adult patients but scaled down in terms of both dose and duration.

The success seen in this case is especially important given the devastating psychological impact of CRPS on children and their entire family. In this specific case, the patient was unable to walk without the use of crutches, spent a significant amount of time crying in pain, and was unable to attend school when she was first seen in our clinic. Early on in treatment, her pain was still so severe it caused her to miss out on a landmark event. She stayed home with her mother while her father and siblings traveled to their home country of Greece. This highlights the impact of pain associated with CRPS on the patient's function and overall quality of life. In this instance the pain not only

\section{REFERENCES}

1. Harden RN, Oaklander AL, Burton AW, Perez RS, Richardson K, Swan M, Barthel J, Costa B, Graciosa JR, Bruehl S. Complex regional pain syndrome: Practical diagnostic and treatment guidelines, 4th edition. Pain Medicine 2013; 14:180-229.

2. Harden RN, Bruehl S, Perez RS, Birklein F, Marinus J, Maihofner C, Lubenow T, Buvanendran A, Mackey S, Graciosa J, Mogilevski M, Ramsden C, Chont M, Vatine JJ. Validation of proposed diagnostic criteria (the "Budapest Criteria") for Complex Regional Pain Syndrome. Pain 2010; 150:268-274.

3. CRPS patients - How many are there in the United States? American RSDHope. www.rsdhope.org/crps---how-many-victimsare-there-in-the-united-states.html. Published 2017. Accessed impacted the child but the child's entire family. Parents of children diagnosed with CRPS have described the process of diagnosis and treatment as the single hardest thing that they've ever encountered. Many watch as their children scream in pain for hours without relief, helpless to do anything about their child's suffering. Having a child who is constantly in pain with symptoms that get worse over time is a devastating situation for parents, leading them to exhaust all resources to obtain the best and most immediate treatment for their child. Epidural drug infusion may just be the treatment option they are seeking.

\section{CONCLUSION}

This case reports describes identification and implementation of an effective multidisciplinary approach which gave a psychologically distraught child her life back when all other physicians had failed. The patient was unable to walk, could not even bend her leg, and was missing out on being a child. The aggressive decision to place a tunneled epidural catheter, which represents uncharted territory in this patient population, is ultimately responsible for the success seen in this patient. She is able to walk and run again - to just be a kid - and her family dynamic is back to what it once was. Had the decision not been made, who knows where the patient would be at this point. Based on this, we suggest considering epidural anesthesia for pain management and to aid in physical and emotional rehabilitation in pediatric CRPS.

\section{Acknowledgments}

The author would like to acknowledge Stephanie Washburn, PhD for her assistance with this case report.
January 27,2018

4. Tan EC, Zijlstra B, Essink ML, Goris RJ, Severijnen RS. Complex regional pain syndrome type I in children. Acta Paediatr 2008; 97:875-879.

5. Rodriguez MJ, Fernandez-Baena M, Barroso A, Yanez JA. Invasive management for pediatric complex regional pain syndrome: Literature review of evidence. Pain Physician 2015; 18:621-630.

6. Zernikow B, Wager J, Brehmer H, Hirschfeld G, Maier C. Invasive treatments for complex regional pain syndrome in children and adolescents: A scoping review. Anesthesiology 2015; 122:699-707. 